\title{
Inverted ILM Flap Technique
}

\author{
Zofia Michalewska, Jerzy Nawrocki
}

Pak J Ophthalmol 2019, Vol. 35, No. 3

As recently as 30 years ago, macular holes were thought to be untreatable. Kelly and Wendel showed that this was not the case in 1991 when they published their pilot study on vitreous surgery for idiopathic macular holes ${ }^{1}$. In 1997, Eckardt et al. introduced internal limiting membrane (ILM) peeling ${ }^{2}$, following which, pars plana vitrectomy, internal limiting membrane peeling, fluid/gas exchange and face-down positioning were soon adopted as the standard treatment method for successful hole closure and BCVA improvement. Surgeons improved their own results by using smaller gauge instruments and, especially, by more qualified patient selection: earlier treatment, non-myopic eyes, better initial visual acuity, and smaller macular holes.

Despite these huge advances, $100 \%$ success rates of the classical approach, briefly described above, that were often quoted in the past were perhaps overly optimistic. Modern OCT-based studies reveal that 19$39 \%$ of cases actually result in "flat open" anatomical outcomes (also referred to in the literature as type two closure), where the margins of the macular hole remain flat with bare retinal pigment epithelium. Although the hole will normally not increase in size, visual acuity does not improve in these cases thus the surgical intervention cannot be said to have been fully successful $^{3}$. OCT images reveal U-Type, V-Type and irregular closure are the three successful macular hole anatomical closure types that also provide better functional improvement.

After careful investigation of OCT images from "flat open" results, Jerzy Nawrocki came to the conclusion that ILM tissue has an actual role to play in ensuring that the macular hole is successfully closed. As a base membrane, its properties enable cell proliferation and reactive gliosis. Inverting the ILM and laying it over the macular hole on the surface of the retina may create a scaffold, over which Müller cells can spread and photoreceptor cells may follow the gliosis, either re-approximating or re-growing over time. The first surgeries with the "Inverted ILM Flap Technique" were carried out between 2006 and 2009 and the highly encouraging results were presented at the American Society of Retinal Specialists (ASRS), European Vitreo-retinal Society (EVRS) and German Ophthalmological Society (DOG) meetings. Our first papers on the inverted flap technique were published in 2009 and $2010^{4,5}$.

The technique itself was modified in 2015 when we introduced the "Temporal Inverted ILM Flap Technique" in order to reduce overall trauma to the nerve fibres thereby reducing the amount of dissociated optic nerve fibre layer (DONFL), described by Tadayoni et $\mathrm{al}^{6}$ and to help prevent the flap moving away from the macular hole during fluid/air exchange ${ }^{7}$. It is a straightforward procedure although the manoeuvres involving manipulating the flap can take some time to perfect. Core vitrectomy is performed and posterior hyaloid detached and removed. Trypan blue is applied for 40 seconds to 1 minute to aid visualisation of the ILM, which is peeled on the temporal side of the macular hole but it is not completely removed. A sufficiently large piece of ILM is left attached at the margins of the hole and turned over and inverted so that the surface previously facing the vitreous body is now facing the retinal pigment epithelium. It is gently massaged over the macular hole (keeping it on top of the retina) and is held in place with fluid/air injection (with the flow moving from the attached end of the ILM flap towards the free end). Patients are asked to maintain a position where the air bubble stays in the centre of their visual field for 3 days.

The flap appears to perform its intended role as a scaffold, as confirmed immunohistochemically by Shiode et $\mathrm{al}^{8}$. Gliosis, migration and proliferation of 
Müller cells, microstructural regeneration, decreased ellipsoid zone defects and the macular hole closing and filling with tissue have all been reported along with BCVA improvements of 1-4 lines - as early as one day after surgery 9 .

Since its introduction, the flap technique has been investigated by many surgeons across the world and various authors have introduced their own adaptations and modifications. These have included filling the hole with attached ILM (which has led to the original techniques proposed by our group being called a "cover" technique and the others "fill" or "stuffing" techniques). Others have used free flaps of unattached ILM, created multiple flaps, transplanted ILM to cover or fill the hole, used a lens capsular flap, or an amniotic membrane flap. Different dyes have been used and alternative methods to hold the flap in place such as viscoelastic and gluconated blood have been reported in the literature ${ }^{10-14}$.

As with any invasive surgical technique and any intervention involving dyes, there are potential risks involved when using the various flap techniques. Comparative studies of the Inverted ILM Flap Technique ("cover" techniques), "fill" techniques and classical ILM peeling seem to show that the Inverted flap technique is a highly effective surgical method to close large macular holes and restore retinal tissue and improve BCVA ${ }^{5,15}$.

Initially developed to deal with large macular holes the inverted flap technique tends to be used more often to treat large, old and myopic macular holes and for reoperations after conventional surgery has failed. However, it has also been adopted to treat routine small cases and has successfully resolved macular hole with retinitis pigmentosa, in an eye with Coat's Disease, (both unpublished) and a case of secondary rupture of a retinal arterial macroaneurysm ${ }^{16}$, as well as to successfully treat persistent macular hole after retinal detachment surgery, macular holes with AMD, traumatic macular holes, diabetic macular holes, macular holes secondary to uveitis and optic pit maculopathy.

SD and SS OCT show foveal microstructure recovery following surgery with the inverted flap technique. Hayashi and Kuriyama found reappearance of the ellipsoid zone and ELM during 6months-follow up (more frequently in large and myopic cases compared to macular hole retinal detachment $)^{17}$. Despite finding expansion of submacular pigment epithelium atrophy during follow-up, Imai and Azumi reported visual acuity improvement 0.08 to 0.3 (Landolt C) ${ }^{18}$. Chen et al. found improvement in multifocal electroretinography after the inverted flap technique for large macular hole and although it was not significantly correlated with BCVA it may be a useful supplement when evaluating functional recovery ${ }^{19}$.

A new macular hole closure type: "flap closure" has recently been described by Bonińska, Nawrocki and Michalewska. In these cases, large macular holes with lower preoperative visual acuity which probably would not have been closable without the use of the inverted flap technique, SD or SS-OCT revealed a thin flap of tissue that had closed the hole one week after surgery. Foveal architecture continuously restored up to one year follow-up and mean visual acuity improved. However, final visual acuity was lower when compared to eyes with initial U- type, V-type or irregular closure ${ }^{20}$.

Whether or not the inverted flap technique should be considered as the first approach for the treatment of all macular holes is still open to discussion. The published outcomes of surgery as well as the comparative studies published to date have meanwhile led to the inverted flap technique and its adaptations being adopted by a significant number of ophthalmic surgeons throughout the world.

\section{REFERENCES}

1. Kelly NE, Wendel RT. Vitreous surgery for idiopathic macular holes. Results of a pilot study. Arch Ophthalmol. 1991; 109: 654-9.

2. Eckardt C, Eckardt U, Groos S, Reale E. Entfernung der Membrana limitans interna bei Makulalöchern Klinische und morphologische Befunde. Ophthalmologe [Article in German], 1997; 4: 545-51.

3. Michalewska Z, Michalewski J, Cisiecki S, Adelman R, Nawrocki J. Correlation between foveal structure and visual outcome following macular hole surgery: a spectral optical coherence tomography study. Graefes Arch Clin Exp Ophthalmol 2008; 246: 823-30.

4. Michalewska Z, Michalewski J, Nawrocki J. Macular hole closure after vitrectomy: The inverted flap technique. Retina Today, 2009: 73.

5. Michalewska Z, Michalewski J, Adelman RA, Nawrocki J. Inverted internal limiting membrane flap technique for large macular holes Ophthalmology, 2010; 117: 2018-25.

6. Tadayoni R, Paques M, Massin P, Mouki-Benani S, Mikol J, Gaudric A. Dissociated optic nerve fiber layer appearance of the fundus after idiopathic epiretinal membrane removal. Ophthalmology, 2001; 108: 2279-83. 
7. Michalewska Z, Michalewski J, Dulczewska-Cichecka K, Adelman RA, Nawrocki J. Temporal inverted internal limiting membrane flap technique versus classic inverted internal limiting membrane flap technique: A comparative study. Retina, 2015; 35 (9): 1844-50.

8. Shiode $\mathbf{Y}$, Morizane $\mathbf{Y}$, Matoba $\mathbf{R}$, Hirano M, Doi S, Toshima S, Takahashi K, Araki R, Kanzaki Y, Hosogi M, Yonezawa T, Yoshida A, Shiraga F. The role of inverted internal limiting membrane flap in macular hole closure. Invest Ophthalmol Vis Sci. 2017; 58: 484755.

9. Michalewska Z, Michalewski J, Dulczewska-Cichecka K, Nawrocki J. Inverted internal limiting membrane flap technique for surgical repair of myopic macular holes. Retina, 2014; 34 (4): 664-9.

10. Nawrocki J, Michalewska Z. SS-OCT for macular hole treated with the inverted internal limiting membrane flap technique. In Nawrocki J, Michalewska Z (ed.): An atlas of SS-OCT. Springer, 2017: p.97-113.

11. Imai H, Azumi A. The expansion of RPE atrophy after inverted ILM flap technique for a chronic large macular hole. Case Rep Ophthalmol. 2014; 5; 5 (1): 83-6.

12. Andrew NA, Chan WO, Tan M, Ebneyter A, Gilhotra JS. Modification of the inverted internal limiting membrane flap technique for the treatment of chronic and large macular holes. Retina, 2016; 36 (4): 834-7.

13. Pires J, Nadal J, Gomes NL. Internal limiting membrane translocation for refractory macular holes. $\mathrm{Br}$ J Ophthalmol. 2017; 101(3):377-382.

Doi:10.1136/bjophthalmol-2015-308299. Epub 2016 May 4.

\section{Author's Affiliation}

Zofia Michalewska, MD, PhD

Ophthalmic Clinic “Jasne Blonia”.

Ul. Rojna 90. Lodz, 91-162, Poland.

Tel: +48 (0)42636 8282 ; Fax: +48 (0)42 6110505

E-mail: zosia_n@yahoo.com

Jerzy Nawrocki

MD, PhD.

Ophthalmic Clinic "Jasne Blonia"

Rojna 90, Lodz, Poland.
14. Dai $\mathbf{Y}$, Dong $F$, Zhang $X$, Yang Z. Internal limiting membrane transplantation for unclosed and large macular holes. Graefes Arch Clin Exp Ophthalmol. 2016; 254 (11): 2095-2099. Epub 2016 Aug 12.

15. Rizzo S, Tartaro R, Barca F, Caporossi T, Bacherini D, Giansanti F. Internal limiting membrane peeling versus inverted flap technique for treatment of full-thickness macular holes: a comparative study in a large series of patients. Retina, 2017; 38 Suppl. 1: S73-S78.

16. Iwakawa $Y$, Imai H, Kaji H, Mori $Y$, Ono C, Otsuka K, Miki A, Oishi M: Autologous Transplantation of the Internal limiting Membrane for Refractory Macular Hole following Ruptured Retinal Arterial Macroaneurysm: A Case Report. Case Rep Ophthalmol. 2018; 1; 9 (1): 113-119. eCollection.

17. Hayashi $\mathbf{H}$, Kuriyama S. Foveal microstructure in macular holes surgically closed by inverted internal limiting membrane flap technique. Retina, 2014; 34 (12): 2444-50.

18. Imai $\mathbf{H}$, Azumi A. The expansion of RPE atrophy after inverted ILM flap technique for a chronic large macular hole. Case Rep Ophthalmol. 2014; 5 (1): 83-6.

19. Chen Z, Zhao C, Ye JJ, Wang XQ, Sui RF. Inverted Internal Limiting Membrane Flap Technique for Repair of Large Macular Holes: A Short-term Follow-up of Anatomical and Functional Outcomes. Chin Med J (Engl). 2016; 129 (5): 511-7.

20. Bonińska K, Nawrocki J, Michalewska Z. Mechanism of "Flap Closure" after the inverted internal limiting membrane flap technique. Retina, 2017; 38 (11): 21842189. 